\title{
BLOCK BUNDLES
}

\author{
BY C. P. ROURKE AND B. J. SANDERSON
}

Communicated by W. Feit, June 15, 1966

Block bundles play the same role in the piecewise linear category, as vector bundles in the differential category. This is an announcement of results supporting this claim, details will appear elsewhere. All maps and spaces are assumed p.l.

A $q$-block bundle $\xi^{q} / K$ consists of a total space $E(\xi)$ and a locally finite simplicial complex $K \subset E(\xi)$, satisfying

(i) For each $n$-simplex $\sigma_{i} \in K \exists$ an $n+q$-ball $\beta_{i} \subset E$ such that $\left(\beta_{i}, \sigma_{i}\right)$ is an unknotted ball pair. $\beta_{i}$ is the block over $\sigma_{i}$.

(ii) $E(\xi)$ is the union of the balls $\beta_{i}$.

(iii) If $\sigma_{i} \cap \sigma_{j}=\sigma_{k}$ (or $\varnothing$ ) then $\beta_{i} \cap \beta_{j}=\beta_{k}$ (or $\varnothing$ ).

Block bundles $\xi^{q}, \eta^{q} / K$ are isomorphic if $\exists$ a homeomorphism $h: E(\xi) \rightarrow E(\eta)$, which preserves the block structure and such that $h \mid K=$ identity.

Now let $K^{\prime}$ be a subdivision of $K$, and suppose given $\xi / K$, then $\xi^{\prime} / K^{\prime}$ is a subdivision of $\xi / K$ if $E(\xi)=E\left(\xi^{\prime}\right)$, and the union of blocks of $\xi^{\prime}$ lying over a simplex $\sigma_{i} \in K$ is the block $\beta_{i}$ of $\xi$. The converse of subdivision is amalgamation.

Let $I_{q}(K)$ be the set of isomorphism classes of $q$-block bundles over $K$, and let $|K|$ denote the polyhedron underlying $K$.

Theorem 1. Suppose $|K|=|L|$ and $J$ a common subdivision. Then the operation of subdivision over $J$, followed by amalgamation induces a bijection

$$
I_{q}(K) \rightarrow I_{q}(L)
$$

which is independent of the choice of $J$.

Now let $X$ be a polyhedron (a space with a related family of locally finite triangulations) and suppose $|K|=|L|=X . \xi / K$ is equivalent to $\eta / L$ if they have isomorphic subdivisions. Denote the set of equivalence classes by $I_{q}(X)$ (isomorphic to $I_{q}(K)$ any $|K|=X$, in a natural way by Theorem 1$)$.

Induced bundles (and Whitney sums) can be defined and $I_{q}($ ) becomes a contravariant homotopy functor on the category of polyhedra. In fact, using an analogue of the Grassmannian we have

Theorem 2. There is a locally finite simplicial complex $B(P L)_{q}$ and a block bundle $\gamma^{q} / B(P L)_{a}$ such that 


$$
T:\left[, B(P L)_{q}\right] \rightarrow I_{q}()
$$

given by $T[f]=f^{*} \gamma^{q}$, is a natural equivalence of functors, (where [, ] denotes homotopy classes of maps).

Block bundles are not fibre bundles, the fibres being replaced by blocks, but they do have associated semisimplicial principal bundles. The s.s. group is $P L_{q}$, a $k$-simplex being an isomorphism of $\varepsilon^{q} / \Delta^{k}$ (the trivial bundle over the standard $k$-simplex) with itself. Boundary operators are induced by restriction. Although degeneracies can be defined in $(P L)_{q}$, the natural choice is not piecewise linear (by the standard mistake) and if modified fails to give homomorphisms. However, $(P L)_{q}$ does satisfy the extension condition and principal bundles can be defined, by omitting mention of degeneracy operations.

Now let $\mathbf{K}$ be the s.s.-complex obtained from $K$ by ordering the vertices (a $k$-simplex is then a monotone simplicial map $\Delta^{k} \rightarrow K$ ).

THEOREM 3. There is a 1-1 correspondence between the set $I_{q}(K)$ and the set of isomorphism classes of principal $(P L)_{q}$-bundles with base $\mathbf{K}$.

Classification of regular neighbourhoods. It is relatively easy to show that if $|K|$ is a manifold and $\xi / K$ a block bundle over $K$, then $E(\xi)$ is also a manifold. In fact, $|K|$ is a proper submanifold of $E(\xi)$ and $E(\xi)$ collapses to $|K|$ so $E(\xi)$ is an abstract regular neighbourhood of $|K|$.

The converse which implies the existence of normal block bundles is

THEOREM 4. Let $M^{n} \subset Q^{n+q}$ be a proper inclusion of a compact submanifold $M$ in an abstract regular neighbourhood $Q$, and suppose $(M, \dot{M})=(|K|,|L|), L$ a subcomplex of $K$.

Then $\exists \xi^{q} / K$ with $\exists(\xi)=Q$.

Moreover, if $U$ is a regular neighbourhood of $\dot{M}$ in $\dot{Q}$ and $\eta^{q} / L$ is such that $E(\eta)=U$, then we can choose $\xi$ so that the restriction $\xi \mid L=\eta$.

The following is an analogue of the tubular neighbourhood theorem

Theorem 5. Suppose $M^{n} \subset V^{n+q}$ a proper submanifold, and $K, L$ as in Theorem 4. Suppose $E\left(\xi^{q} / K\right), E\left(\zeta^{q} / K\right)$ are regular neighbourhoods of $M$ in $V$, which meet $\dot{V}$ regularly i.e. in $E(\xi \mid L), E(\zeta \mid L)$ respectively.

Then $\exists$ an isotopy of $V \bmod M$ (i.e. keeping $M$ pointwise fixed) realising an isomorphism $\xi \cong \zeta$.

Moreover, if $\xi|L=\zeta| L$ then we can choose to isotope mod $\dot{V}$.

REMARK. The proofs of Theorems 4 and 5 use little more than the 
relative regular neighbourhood theorems of Hudson and Zeeman [4].

Now let $\mathscr{K}_{q}\left(M^{n}\right)$ denote equivalence classes of $n+q$-dimensional abstract regular neighbourhoods of $M$ (where the equivalence is homeomorphism $\bmod M$ ). We have from Theorems 2,4 and 5 (a similar theorem can be found in the works of Haefliger, to appear),

THEOREM 6. There is a bijection

$$
R:\left[M, B(P L)_{q}\right\rfloor \rightarrow \mathscr{N}_{q}(M) .
$$

Transversality. There are analogues of the smooth transversality theorems (cf. also Armstrong and Zeeman [1]).

Let $N, M \subset Q$ be proper compact submanifolds and $\xi$ a blocked regular neighbourhood of $M$ in $Q$. We write $N \uparrow \xi M$ if $\exists$ a subdivision $\xi^{\prime}$ of $\xi$ s.t. $E\left(\xi^{\prime} \mid N \cap M\right)=E(\xi) \cap N$, and $N \perp_{\xi} M$ if this is true near $M$, i.e. $\exists$ a neighbourhood $U$ of $M$ in $Q$ and $\xi^{\prime}$ a subdivision of $\xi$ s.t. $E\left(\xi^{\prime} \mid N \cap M\right) \cap U=E(\xi) \cap N \cap U$.

Theorem 7. Suppose $\xi, N, M, Q$ are as above. Then $\exists$ an $\epsilon$-isotopy of $Q$ carrying $N_{\xi} \perp M$.

Moreover, if $\dot{N} \Lambda_{\xi \mid \dot{M}} \dot{M}$, then we can choose to isotope $\bmod \dot{Q}$.

TheOREM 8. As in Theorem 7 and $\xi$ meeting $\dot{Q}$ regularly. Then $\exists$ an isotopy of $Q$ carrying $N \perp_{\xi} M$, and if $\dot{N} \perp_{\xi \mid \dot{M} \dot{M}}$ we can choose to isotope $\bmod \dot{Q}$.

Classification of smoothings. A piecewise differentiable analogue of $(P L)_{q},(P D)_{q}$ can be defined, so that the orthogonal group acts on the left, and (cf. Lashof and Rothenberg [5]), we have

THEOREM 9. There is an s.s.-fibration

$$
(P D)_{q} / O_{q} \rightarrow B O_{q} \rightarrow B(P L)_{q}
$$

where $B_{q}, B(P L)_{q}$ are s.s.-classifying spaces for $O_{q},(P L)_{q}$ respectively.

Now let $M^{n} \subset Q^{n+q}$ be a proper submanifold (both compact) and let $Q_{\alpha}^{n+q}$ denote $Q$ together with a smoothing $\alpha$ (i.e. a compatible differential structure). Let $\Gamma\left(M, Q_{\alpha}\right)$ denote the set of equivalence classes of smoothings of $Q$ (concordant to $\alpha$ on $Q$ ), under the equivalence of concordance such that $M$ is a smooth submanifold throughout the concordance.

Combining our results on regular neighbourhoods with Theorem 9 and the main result of [5] (cf. also Haefliger and Wall [2] for a similar theorem) we have 
THEOREM 10. The smoothing $\alpha$ determines a bijection between $\Gamma\left(M, Q_{\alpha}\right)$ and the set of homotopy classes of sections of a fibration, with fibre $(P D)_{a} / O_{q}$, associated with the principal $(P L)_{a}$-bundle corresponding to a regular neighbourhood of $M$ in $Q$.

Suppose $M$ and $Q$ are spheres, the inclusion and smoothing standard, and denote the resulting set $\Gamma_{n}^{q}$. The following corollary, which is a desuspension of a result of Hirsch [3], can also be proved directly using the methods of [3].

Corollary 11. $\Gamma_{n}^{q} \cong \pi_{n}\left((P D)_{q}, O_{q}\right)$.

Microbundles. There is also a theory of micro-block bundles which, as is easily proved, coincides with the standard theory (given here). It may be that the theory of microbundles à la Milnor coincides with the theory of block bundles. This is true in the stable range, $q>n+1$, but in general, although a microbundle gives rise to a unique equivalence class of block bundles, there is an obstruction theory for the converse, with coefficients in $\pi_{n}\left((P L)_{q}, P L_{q}\right)$. The crucial question in this respect is

Question. Let $\Sigma^{n} \subset \Sigma^{n+q}$ be the standard sphere pair. Does the embedding have a normal microbundle which cannot be isotoped onto the standard micro normal bundle for some $n, q$ ?

Added in proof. Similar results have been announced by C. Morlet, Les voisinages tubulaires des variêtés semi-linéaires, C. R. Acad. Sci. Paris 262 (1966), 740-743.

\section{REFERENCES}

1. M. A. Armstrong and E. C. Zeeman, Transversality for piecewise linear manifolds, (to appear)

2. A. Haefliger and C. T. C. Wall, Piecewise linear microbundles in the stable range, (preprint).

3. M. W. Hirsch, Obstruction theories for smoothing manifolds and maps, Bull. Amer. Math. Soc. 69 (1963), 352-356.

4. J. F. P. Hudson and E. C. Zeeman, On regular neighbourhoods, Proc. London Math. Soc. 14 (1964), 714-745.

5. R. Lashof and M. Rothenberg, Microbundles and smoothing, Topology 3 (1965), $357-388$.

Queen Mary College, London and UNIVERSITY OF WARWICK, WARWICKSHIRE 